\title{
Fatores determinantes da retenção de peso no pós-parto: uma revisão da literatura
}

\author{
Determinants of postpartum weight gain \\ retention: a literature review
}

\footnotetext{
1 Departamento de Nutrição Social e Aplicada, Instituto de Nutrição Josué de Castro, Universidade Federal do Rio de Janeiro. Av. Brigadeiro Trompowsky $s / n o$, bloco J, 2o andar, Rio de Janeiro, $R J$ 21941-590, Brasil. gkac@gbl.com.br 2 Faculdade de Saúde Pública, Universidade de São Paulo. Av. Dr. Arnaldo 715, São Paulo, SP 01246-904, Brasil.
}

\begin{abstract}
This study is a literature review of factors determining postpartum weight gain retention. According to the literature, the main factors include gestational weight gain and intensity of lactation. Various studies show that the greater the weight gain during pregnancy, the greater the postpartum weight retention. Smoking was associated with lower retention, while lactation only demonstrated a protective effect against weight retention in some studies. Various factors, like physical activity, food consumption, age at menarche, number of abortions, and mother's work status have still not been properly studied. The conclusion is that there are few studies on postpartum weight changes, suggesting the need for new research on the theme, especially in developing countries where obesity has been insufficiently researched thus far.
\end{abstract}

Key words Obesity; Weight Gain; Body Weight; Prospective Studies

Resumo Este trabalho constitui-se em uma revisão de literatura sobre fatores determinantes da retenção de peso em mulheres no pós-parto. Os resultados da revisão demonstraram que os principais fatores determinantes incluem o ganho de peso gestacional e a intensidade da lactação. Diversos estudos demonstraram que quanto maior o ganho de peso durante a gestação, maior a retenção de peso no pós-parto. O fumo esteve associado a uma menor retenção, enquanto a lactação só exerceu efeito protetor para a retenção de peso em alguns estudos. Diversos fatores, como a atividade física, o consumo de alimentos, a idade de menarca, número de abortos e o trabalho materno, ainda não foram devidamente estudados. Conclui-se que são escassos os estudos sobre mudanças de peso no pós-parto, o que sugere a realização de novos trabalhos sobre o tema, especialmente em países em desenvolvimento onde a obesidade ainda é pouco estudada.

Palavras-chave Obesidade; Ganho de Peso; Peso Corporal; Estudos Prospectivos 


\section{Introdução}

Verifica-se atualmente a importância da obesidade como problema emergente de saúde pública em diversos grupos etários e em populações do mundo todo. No Brasil, o problema é particularmente importante em mulheres de grupos sócio-econômicos menos privilegiados (INAN, 1991). Sobal \& Stunkard (1989), em uma ampla revisão, demonstraram que os determinantes da obesidade são diversos e incluem hábitos alimentares inadequados, sedentarismo, alta paridade, entre outros. Estes fatores seriam potencializadores da suscetibilidade genética.

Rossner \& Ohlin (1995) identificaram a idade e o número de gestações como fatores de risco para o desenvolvimento de sobrepeso e obesidade, sendo estes, portanto, importantes na determinação do problema em mulheres em idade reprodutiva. Em uma recente revisão de literatura, Gunderson \& Abrams (1999) demonstraram que o ganho de peso durante a gestação e diversos outros fatores exercem influência nas mudanças de peso no pós-parto e no desenvolvimento da obesidade. Essa revisão revelou ainda que são escassos os estudos sistemáticos com mulheres no pós-parto, e, entre os já realizados, quase todos apresentaram, pelo menos, uma limitação metodológica. Algumas características do estilo de vida, como o hábito de fumar, o consumo de álcool, a atividade física e a história obstétrica, não têm sido devidamente estudadas em forma conjunta.

A presente revisão tem como principal objetivo descrever os determinantes da retenção de peso e da obesidade em mulheres no pósparto. Para cumprir tal finalidade, organiza-se em diversas seções. Inicialmente, são apresentados dados sobre a tendência do sobrepeso e da obesidade na população brasileira. Nas seções seguintes, são discutidos os determinantes da retenção de peso em mulheres no pósparto. $\mathrm{O}$ papel dos fatores relacionados à gestação, com ênfase na paridade, e o dos relacionados ao pós-parto, com ênfase na lactação, são apresentados. Em seguida, são discutidas algumas questões metodológicas relacionadas aos estudos sobre determinantes da retenção de peso no pós-parto, e na seção final são apresentadas as conclusões.

Em relação à definição da variável dependente (retenção de peso), vale um comentário. Na realidade, o que está sendo estudado são mudanças de peso no pós-parto, no entanto a situação mais comum e de implicação sanitária é a retenção de peso. Dessa forma, utiliza-se essa terminologia em grande parte da revisão.

\section{A magnitude do sobrepeso e da obesidade no Brasil}

Contrariamente ao evidenciado nos Estados Unidos e em alguns países europeus, no Brasil, a realização de inquéritos antropométricos de representatividade nacional não ocorre de forma sistemática. Assim, os dados disponíveis referem-se ao Estudo Nacional de Despesas Familiares (ENDEF) realizado entre 1974-1975; à Pesquisa Nacional sobre Saúde e Nutrição (PNSN), de 1989; mais recentemente, à Pesquisa Nacional sobre Demografia e Saúde (PNDS), realizada em 1996; e à Pesquisa sobre Padrão de Vida (PPV), de 1997 (BEMFAM, 1997; INAN, 1991; Monteiro, 1999; Monteiro \& Conde, 1999).

Entre 1974-1975 e 1989, a freqüência de sobrepeso/obesidade em mulheres maiores de 18 anos (Índice de Massa Corporal (IMC) $\geq 25,0$ ) aumentou de $25,8 \%$ para $38,1 \%$. Observou-se um aumento de $40 \%$ no sobrepeso I (IMC 25,0 a 29,9 ) e de quase $70 \%$ para o sobrepeso II e III (IMC $\geq 30,0$ ) (INAN, 1991).

Outros resultados reportados pela PNSN 1989 revelaram que a Região Sul apresentou as maiores prevalências de sobrepeso (34\% nos homens e $43 \%$ nas mulheres), sendo estas semelhantes e até superiores as de alguns países desenvolvidos. A PNSN ainda demonstrou que o problema de sobrepeso/obesidade era basicamente feminino, já que $12 \%$ das mulheres apresentaram este problema em comparação com uma prevalência de $4,8 \%$ observada entre os homens. Outra constatação interessante e surpreendente foi o fato de que a prevalência de sobrepeso/obesidade atingiu $25,8 \%$ das mulheres de baixa renda, achado pouco comum em países desenvolvidos (INAN, 1991; Sichieri et al., 1994).

Estimativas recentes, baseadas na PNDS realizada em 1996, revelaram um aumento de $10 \%$ para o sobrepeso I e $20 \%$ para o sobrepeso II + III. Apesar de os aumentos nas prevalências de sobrepeso terem sido observados no país como um todo, o fenômeno foi mais marcante na área rural do Norte/Nordeste, onde a prevalência de sobrepeso II + III quase dobrou no período entre as duas pesquisas, passando de $3,2 \%$ para $6,1 \%$ (Coitinho, 1998).

A tendência na prevalência da obesidade (IMC $\geq 30,0$ ) para a população brasileira foi muito bem sintetizada em recente publicação sobre a epidemiologia da obesidade (Monteiro, 1999). Segundo dados do ENDEF e da PNSN, a prevalência de obesidade aumentou 0,36 ponto percentual ao ano para as mulheres e 0,20 para os homens, entre 1975 e 1989. Esses valores são inferiores aos observados para a popu- 
lação masculina do Kuwait e feminina da Rússia, e similares aos da população masculina da Alemanha (Monteiro, 1999).

A tendência contemporânea para a obesidade foi analisada somente para a população feminina, já que a PNDS coletou dados de peso e estatura apenas em mulheres em idade reprodutiva, com pelo menos um filho menor de cinco anos. Os dados do ENDEF e da PNSN foram então novamente analisados segundo esse critério e revelaram que, entre 1975 e 1989, a tendência de aumento na prevalência de obesidade foi de 0,20 ponto percentual ao ano, enquanto, para o período 1989-1996, a tendência foi de 0,37 (Monteiro, 1999).

Os dados mais recentes sobre a prevalência da obesidade provêm da Pesquisa sobre Padrão de Vida (PPV) realizada em 1997 pelo IBGE apenas para as regiões Nordeste e Sudeste. Segundo dados de Monteiro \& Conde (1999), a prevalência de obesidade aumentou em ambas as regiões na população masculina, todavia o aumento foi bem maior (95\%) para a Região Nordeste quando comparado ao da Região Sudeste (38\%). Entre as mulheres da Região Nordeste, o aumento na prevalência de obesidade foi de $60 \%$; por outro lado, a prevalência para as mulheres da região Sudeste diminuiu ligeiramente (resultados não significativos).

Um achado particularmente interessante da PPV foi o declínio na prevalência de obesidade observado entre as mulheres da Região Sudeste do maior quartil de renda. A queda de 13,2\% em 1989 para 8,2\% em 1997 não foi até o momento observada em nenhum país em desenvolvimento, sendo considerado evento singular (Monteiro \& Conde, 1999).

\section{Determinantes da retenção de peso e da obesidade em mulheres}

Diversos fatores têm sido investigados e relacionados à ocorrência de sobrepeso/obesidade em mulheres. Estes incluem variáveis relacionadas à história reprodutiva, sobretudo à paridade (Arroyo et al., 1995; Billewicz \& Thomson, 1970; Brown et al., 1992; Coitinho, 1998; Heliovara \& Aromaa, 1981; Newcombe, 1982), à intensidade da lactação (Barbosa et al., 1997; Butte \& Hopkinson, 1998; Dewey et al., 1993; Kramer et al., 1993; Potter et al., 1991), à atividade física (Boardley et al., 1995; Dewey, 1998a, 1998b; Lovelady et al., 2000), assim como fatores ligados ao estilo de vida no pós-parto (Leermakers et al., 1998; Ohlin \& Rossner, 1990, 1996; Parham et al., 1990; Rossner, 1992; Sampselle et al., 1999; Schauberger et al., 1992; Smith et al., 1994).
Os principais fatores determinantes para retenção de peso e para a obesidade no pósparto são discutidos nesta seção, tendo como base a literatura internacional produzida sobre o assunto nos últimos trinta anos, em especial a década de 90 .

\section{Fatores relacionados à gestação}

\section{Paridade}

O primeiro estudo sobre a ocorrência de obesidade associada à gestação foi realizado por Sheldon \& London (1949). Esses autores apontaram para a possibilidade de uma mulher desenvolver obesidade após o parto e sugeriram que mulheres multíparas que eram obesas ganhavam peso de forma cadenciada, e não subitamente, em associação a uma gestação específica.

McKeown \& Record (1957) estudaram a evolução do peso desde a primeira consulta prénatal até 24 meses após o parto em 1.118 mulheres com filhos nascidos entre abril de $1949 \mathrm{e}$ março de 1950. A dinâmica da evolução do peso no pós-parto indicou que entre a concepção e três meses depois do nascimento houve uma retenção média de $3,58 \mathrm{~kg}$. Considerando o período entre a concepção e 24 meses após o parto, a retenção de peso foi de 3,04kg. Em relação à paridade, os autores observaram uma maior retenção média de peso 24 meses após o parto com o aumento do número de filhos. A retenção foi de 3,09kg, 3,44kg e 3,58kg para mulheres com a primeira, segunda e terceira gestações, respectivamente.

Os estudos associando a paridade e o ganho de peso em mulheres ganham intensidade na década de 70 com as investigações realizadas por Abitbol (1969) e Billewicz \& Thomson (1970). Neste último estudo, os autores observaram que, apesar de presente, o efeito da paridade no ganho de peso foi pequeno, depois de controlado para os efeitos da idade e da tendência secular no aumento do peso. Por outro lado, a proporção de mulheres com altos incrementos no peso aumentou com a paridade, mesmo após os ajustes para idade e tendência secular. Por fim, Billewicz \& Thomson (1970) concluíram que, na ausência de efeitos significativos da paridade no peso, a gordura corporal acumulada durante a gestação seria, em sua quase totalidade, perdida entre gestações.

No início da década de 80, grandes estudos começaram a ser realizados e a paridade passou a ser sistematicamente associada à obesidade. Um importante exemplo dessa década 
foi a investigação realizada por Heliovara \& Aromaa (1981) em uma amostra de mais de 17 mil mulheres finlandesas entre 25 e 84 anos. De uma forma geral, a média de IMC aumentou consistentemente com a idade, especialmente nas mulheres entre 24 e 35 anos. Após o ajuste para a idade, os autores concluíram que mulheres com alta paridade (dez ou mais filhos) eram em média $2,3 \mathrm{~kg} / \mathrm{m}^{2}$ mais pesadas do que as nulíparas. A prevalência de obesidade, medida pelo IMC $>30,0 \mathrm{~kg} / \mathrm{m}^{2}$, também esteve relacionada com a paridade.

Após a idade, a paridade foi o mais potente fator preditor da obesidade, apesar dos baixos valores de seus coeficientes parciais de correlação, indicando que essa variável faz parte de um elenco de determinantes da obesidade em mulheres.

Outro estudo realizado no período foi o de Newcombe (1982), com uma amostra representativa de 35.556 mulheres de Cardiff. O autor estimou taxas de ganho de peso para idade, paridade, classe social e fumo. Os resultados revelaram que a taxa de incremento de peso relacionou-se de forma independente com a idade e a paridade, apesar de os dois efeitos estarem fortemente confundidos. Por outro lado, o autor observou que a paridade apresentou um efeito independente da ordem de $0,7 \mathrm{~kg}$ para cada filho.

Já na década de 90, três investigações destacam-se no estudo da associação entre paridade, mudanças de peso e obesidade em mulheres.

O estudo desenvolvido por Brown et al. (1992) investigou a relação entre IMC médio, idade e paridade em um grupo de $41.184 \mathrm{mu}$ lheres entre 55 e 69 anos que responderam a um questionário sobre a associação entre IMC, distribuição de gordura e incidência de câncer. Os valores de IMC foram calculados de forma retrospectiva para as idades 18, 30, 40 e 50 anos, com base em dados reportados de peso e estatura. O sobrepeso foi definido como IMC $>27$ $\mathrm{kg} / \mathrm{m}^{2}$, e a paridade, como o número de filhos vivos reportados.

As mulheres com mais de três filhos apresentaram peso médio, valores de IMC e prevalência de obesidade maiores que as demais em todas as idades examinadas. A paridade explicou $3 \%$ do aumento do peso na faixa etária entre 18 e 50 anos para mulheres com um único filho, percentual que aumentou para $31 \%$ para mulheres com nove filhos ou mais. As análises de regressão entre mudanças de peso e paridade indicaram um ganho de $0,55 \mathrm{~kg}$ por filho vivo nas idades entre 18 e 50 anos. Por outro lado, verificou-se que a influência da idade sobre o ganho de peso foi marcante.
Um dos poucos estudos sobre o tema realizado em países em desenvolvimento foi o de Arroyo et al. (1995). Nesse estudo, foram pesquisadas 1.012 mulheres mexicanas entre $14 \mathrm{e}$ 48 anos de idade, pertencentes a classes de renda média e baixa. As conclusões são semelhantes às observadas por Brown et al. (1992), ou seja, a de que existe uma associação independente entre paridade, idade e prevalência de sobrepeso e/ou ganho de peso em mulheres em idade reprodutiva.

O maior efeito da paridade na prevalência de sobrepeso foi observado nos grupos de mulheres menores de 24 e entre 25 e 34 anos. Nesses grupos, a prevalência aumentou de 19,5\% para $37,7 \%$ e de $28,3 \%$ para $53,2 \%$, quando comparadas mulheres com menos de duas gestações e aquelas com duas ou mais, respectivamente. Por fim, os resultados de uma regressão logística tendo o sobrepeso como variável dependente demonstraram a presença de interação entre os dois fatores. As mulheres com duas ou mais gestações e com mais de 35 anos apresentaram oito vezes mais chance de desenvolver obesidade. Esses valores foram seis vezes mais elevados quando apenas a idade foi considerada e três vezes quando apenas a paridade foi o fator analisado.

A investigação conduzida por Ohlin \& Rossner (1990) em 1.423 mulheres suecas faz parte de um grande estudo longitudinal sobre o monitoramento do peso durante a gestação e no período pós-parto, assim como seus fatores determinantes. Em relação à idade e à paridade, esses autores observaram que as mudanças de peso estavam mais associadas à idade do que à paridade, observações semelhantes às de Billewicz \& Thompson (1970) e de Rookus et al. (1987).

De uma forma geral, os estudos que têm enfocado a relação entre mudanças de peso na idade reprodutiva e os efeitos da idade e da paridade são contraditórios. Algumas pesquisas têm demonstrado incrementos médios que variam de $0,5 \mathrm{~kg}$ a $2,3 \mathrm{~kg}$, controlando para o efeito da idade (Arroyo et al., 1995; Billewicz \& Thompson, 1970; Brown et al., 1992; Heliovara \& Aromaa, 1981; Ohlin \& Rossner, 1990; Parker \& Abrams, 1993), enquanto outros não reportam efeito da paridade (Rookus et al., 1987). A análise desses trabalhos permitiu evidenciar, por outro lado, que o efeito independente da idade foi maior quando comparado ao da paridade. 


\section{Ganho de peso gestacional}

As recomendações sobre o ganho de peso durante a gestação têm sistematicamente sido alvo de debates. O Institute of Medicine dos Estados Unidos, em sua última publicação (IM, 1990), recomendou que o ganho de peso durante a gestação fosse diferenciado segundo o estado nutricional pregresso da mulher. Dessa forma, mulheres com valores de IMC entre 19,9 e 26 deveriam ganhar de $11,4 \mathrm{~kg}$ a $15,9 \mathrm{~kg}$, enquanto, em mulheres com sobrepeso (IMC $\geq$ $26,1 \leq 29$ ), o ganho deveria ser entre $6,8 \mathrm{~kg}$ e $11,4 \mathrm{~kg}$.

Segundo Keppel \& Taffel (1993), existe atualmente uma tendência de aumento em relação às recomendações de ganho de peso durante a gestação, motivadas, sobretudo, por preocupações com a saúde da criança. Por outro lado, somente recentemente surgiu a preocupação com a retenção de peso após o parto, o que pode fazer com que as recomendações de ganho de peso sejam pouco atraentes para algumas mulheres.

O ganho de peso durante a gestação tem sido descrito como um dos mais importantes determinantes para a retenção de peso no pósparto. A literatura sobre o tema é bastante concordante e tem sistematicamente reportado que quanto maior o ganho ponderal, maior a retenção de peso no pós-parto.

Dados do estudo de Ohlin \& Rossner (1990) revelaram que mulheres com ganhos de peso correspondentes ao percentil $90(16,5 \mathrm{~kg})$ retiveram $3,3 \mathrm{~kg}(3,9 \mathrm{DP})$ um ano depois do parto, enquanto mulheres situadas no percentil $10 \mathrm{da}$ distribuição de ganho ponderal $(7,5 \mathrm{~kg})$ não retiveram peso (média $=0,0 \mathrm{~kg}, 3,3 \mathrm{DP}$ ). Resultados de uma regressão linear demonstraram que o ganho ponderal durante a gestação explicou $8 \%$ do peso retido após o parto.

O estudo conduzido por Greene et al. (1988) considerou a relação entre mudanças de peso entre duas gestações segundo o ganho de peso na primeira gestação. Os dados de peso prégestacional foram reportados e os ganhos de peso aferidos durante a gestação. Na média, mulheres que ganharam $13,6 \mathrm{~kg}$ durante a primeira gestação foram $2,7 \mathrm{~kg}$ mais pesadas no início da gestação seguinte. Os autores concluíram que o ganho de peso acima das recomendações foi um importante fator para a retenção de peso.

Um aspecto metodológico importante foi o fato de os autores terem introduzido o intervalo interpartos como variável de controle, o que acabou por atenuar parcialmente o efeito do ganho de peso em virtude da idade, que teria ocorrido no período caso as mulheres não estivessem grávidas (Greene et al., 1988).

De toda a revisão, apenas um artigo apresentou como foco central a relação entre retenção de peso e intervalo interpartos. Sowers et al. (1998) compararam mudanças no peso em um grupo de mulheres que amamentaram até seis meses e com intervalo interpartos de 18 meses (casos) com mulheres que também amamentaram até seis meses, mas não tiveram uma gravidez subseqüente (controles). Não se observou diferença no padrão de retenção de peso entre os grupos, no entanto os casos foram $1,3 \mathrm{~kg}$ mais pesados do que na gravidez inicial (basal), sendo o maior preditor desse aumento de peso as mudanças no peso durante o intervalo interpartos (Sowers et al., 1998).

Os dados reportados por Shauberger et al. (1992) também revelaram um efeito importante do ganho de peso durante a gestação sobre a retenção de peso no pós-parto. Nesse estudo, mulheres com ganhos superiores a $16 \mathrm{~kg}$ foram $5 \mathrm{~kg}$ mais pesadas seis meses após o parto, quando comparadas a mulheres que ganharam menos de $11,3 \mathrm{~kg}$.

No estudo conduzido por Scholl et al. (1995), os autores observaram que mulheres com ganho excessivo de peso, definido como valor superior a $0,68 \mathrm{~kg} / \mathrm{semana}$, entre a $20 \underline{a}$ e a $36 \underline{a}$ semana de gestação, apresentaram maior retenção de peso e atingiram valores de IMC mais elevados no pós-parto, quando comparadas com mulheres com taxas inferiores a $0,68 \mathrm{~kg} /$ semana.

Uma série de outros estudos também revelou importante contribuição do ganho de peso na retenção de peso no pós-parto (Boardley et al., 1995; Parker \& Abrams, 1993; Potter et al., 1991). Um aspecto relevante no âmbito epidemiológico refere-se ao fato de que parcelas consideráveis de mulheres ganham mais peso do que o recomendado. Como exemplo, podemos citar o estudo de Parham et al. (1990), no qual $28 \%$ das mulheres ganharam mais do que os $15,9 \mathrm{~kg}$ recomendados pelo Instituto de Medicina (IM, 1990).

Um aspecto importante refere-se à identificação do momento em que o ganho de peso é maior durante a gestação. Segundo dados de Muscati et al. (1996), o ganho até a vigésima semana foi um importante preditor da retenção de peso no pós-parto para 371 mulheres brancas. O ganho de peso durante a gestação explicou $65,1 \%$ da variabilidade da retenção de peso no pós-parto nesse grupo de mulheres. 


\section{Fatores relacionados ao pós-parto}

\section{Mudanças no estilo de vida}

Diversos fatores ligados ao estilo de vida influenciam nas mudanças de peso no pós-parto (Lederman, 1993). Pode-se considerar que em torno de $15 \%$ das mulheres fumantes o deixam de fazer quando engravidam. Ohlin \& Rossner (1990) verificaram que mulheres que pararam de fumar no começo da gravidez ganharam mais peso $(16,1 \mathrm{~kg})$ do que as não fumantes $(13,9$ $\mathrm{kg}$ ) ou fumantes persistentes $(13,8 \mathrm{~kg})$. Considerando o peso pré-gestacional reportado, estes mesmos autores observaram que o peso retido um ano após o parto foi maior para ex-fumantes $(3,4 \mathrm{~kg})$, intermediário para não fumantes $(1,5 \mathrm{~kg})$, sendo o mais baixo para as mulheres que não pararam de fumar $(0,9 \mathrm{~kg})$.

Schauberger et al. (1992) também observaram que o peso retido seis meses após o parto em não fumantes $(1,7 \mathrm{~kg})$ foi maior do que em mulheres que não pararam de fumar $(0,6 \mathrm{~kg})$. Mulheres fumantes apresentaram maior perda de peso seis meses depois do parto $(13,5 \mathrm{~kg}) \mathrm{em}$ comparação com não fumantes $(11,9 \mathrm{~kg})$, contudo, com duas e seis semanas após o parto, as diferenças entre fumantes e não fumantes não foram estatisticamente significativas.

Alguns estudos têm enfocado a relação entre atividade física, dieta e retenção de peso no pós-parto (Boardley et al., 1995; Dewey, 1998a, 1998b; Ohlin \& Rossner, 1994; Schauberger et al., 1992).

Ohlin \& Rossner (1994) reportaram uma correlação negativa entre retenção de peso no pós-parto (7 a 12 meses) e grau de atividade física $(r=-0,05, p<0,05)$ para 1.423 mulheres suecas. Os autores, por outro lado, discutem que nem os hábitos alimentares, nem o grau de atividade física puderam ser determinados como preditores para a retenção de peso. Na realidade, a única tendência evidenciada, e mesmo assim não significativa, foi a de mulheres com ocupações com altas demandas energéticas apresentarem uma maior retenção de peso. Isso, segundo os autores, poderia ser explicado por uma diminuição do gasto energético durante e depois da gestação, quando comparado ao despendido durante o trabalho.

Boardley et al. (1995) investigaram o efeito de uma série de fatores nas mudanças de peso no pós-parto (7 a 12 meses) em 335 mulheres participantes de um programa de suplementação alimentar. A atividade física no pré e pósparto foi reportada, todavia apenas o grau de atividade física anterior à gestação esteve associado com a retenção de peso no pós-parto. Es- se efeito diferiu segundo a raça e foi considerado no modelo final como um termo de interação. Dessa forma, para mulheres brancas, quanto maior a atividade física reportada antes da gestação, maior a retenção de peso no pósparto. Comparações entre o maior e o menor quartil de atividade física revelaram uma diferença na retenção de peso de quase $1 \mathrm{~kg}$.

Dewey (1998b), com base nos resultados de um estudo de intervenção randomizado, recomenda a prática de exercício físico e o controle dietético como parte de qualquer programa para redução de peso no pós-parto. A autora aponta, ainda, que a prática do exercício físico não apresenta efeitos adversos na produção de leite.

Na mesma linha do estudo de Dewey (1998b), Lovelady et al. (2000), valendo-se de um ensaio clínico randomizado, concluíram que um programa moderado de exercício físico, em conjunto com uma leve restrição calórica, é benéfico na perda de peso em mulheres lactantes com sobrepeso. Os autores concluem que uma perda entre $1 \mathrm{~kg}$ e $2 \mathrm{~kg}$ por mês não compromete a produção de leite e, conseqüentemente, o crescimento infantil (Lovelady et al., 2000).

Os resultados de Sampselle et al. (1999) também revelaram efeito positivo da atividade física no pós-parto. Segundo esses autores, aproximadamente $35 \%$ das mulheres estudadas reportaram realizar atividade física vigorosa pelo menos três vezes por semana. As mulheres mais ativas retiveram menos peso $(3,9$ $\mathrm{kg}$ ) do que as menos ativas $(5,1 \mathrm{~kg})$, o que indica a importância de um programa bem elaborado de atividade física no pós-parto.

Em um outro estudo de intervenção em mulheres no pós-parto, Leermakers et al. (1998) estudaram noventa mães com, pelo menos, 6,8 kg de excesso em relação ao peso pré-gestacional. As mulheres foram alocadas aleatoriamente em dois grupos. O primeiro incluiu um programa por correspondência de perda de peso durante seis meses e o segundo não recebeu tratamento (grupo controle). As mulheres foram avaliadas em relação ao peso corporal, atividade física e padrão alimentar, antes e depois do tratamento. Os resultados demonstraram que as mães que receberam tratamento perderam mais peso $(7,8 \mathrm{~kg})$ em comparação ao grupo-controle $(4,9 \mathrm{~kg}, \mathrm{p}<0,03)$, assim como também perderam um maior percentual do excesso de peso do pós-parto ( $79 \%$ x 44\%, p < 0,01). Os autores concluíram que a implementação de um programa por correspondência de perda de peso pode ser efetivo na redução da retenção de peso no pós-parto.

Outros fatores relacionados ao estilo de vida envolvem o retorno ao trabalho e mudanças 
na auto-imagem. Greene et al. (1988), por exemplo, verificaram que mulheres que retornaram ao trabalho duas semanas após o parto retiveram apenas $0,3 \mathrm{~kg}$ seis meses após o parto, em comparação com $2,1 \mathrm{~kg}$ entre mulheres que não retornaram ao trabalho. Segundo Lederman (1993), quase 50\% das mães americanas permanecem desempregadas durante o primeiro ano do pós-parto e elas acabam permanecendo mais tempo em contato com alimentos, o que não favorece a perda de peso.

\section{Lactação}

Para introduzir a discussão sobre mudanças na composição corporal no período reprodutivo, e em especial durante a lactação, é oportuno apresentar os postulados sugeridos por Robinson (1986). Segundo esse autor, as mudanças que ocorrem na composição corporal caracterizam-se, em primeiro lugar, pela diversidade. Em uma série de espécies, inclusive o homem, parece existir um mecanismo antecipatório de preparação, no qual ocorre depósito de gordura durante a gestação, para posterior utilização durante a lactação. Essas mudanças são respostas a uma seqüência complexa de estímulos neuroendócrinos e bioquímicos que se iniciam com a concepção e sofrem modificações impostas por limitações do ambiente.

Apesar de os postulados de Robinson (1986) indicarem uma relação entre lactação e mudanças na composição corporal, os achados dos estudos sobre o efeito da lactação na retenção de peso no pós-parto têm sido bastante controvertidos.

Em relação ao tipo de alimentação infantil, os achados são contraditórios. Diversos estudos não verificaram maior perda de peso ou gordura corporal entre mães que amamentaram, quando comparadas às que alimentaram seus filhos com formulados lácteos (Brewer et al., 1989; Dugdale \& Eaton-Evans, 1989; Manning-Dalton \& Allen, 1983; Naismith \& Ritchie, 1975; Potter et al., 1991; Schauberger et al., 1992). Por outro lado, somente alguns estudos reportaram efeito protetor da lactação sobre a retenção de peso pós-parto (Dewey et al., 1993; Greene et al., 1988; Janney et al., 1997; Kramer et al., 1993; Ohlin \& Rossner, 1990), enquanto apenas um reportou ganho de peso (Rookus et al., 1987).

Brewer et al. (1989) realizaram um dos primeiros estudos mais consistentes sobre o efeito da amamentação na retenção de peso e gordura corporal no pós-parto. Os autores investigaram 56 mulheres em três ocasiões: com um a dois dias, três e seis meses após o parto. As mães foram categorizadas em três grupos segundo o método de alimentação infantil: aquelas que amamentaram de forma exclusiva, as que utilizaram alimentos formulados exclusivamente e as que combinaram os dois métodos. Os resultados não revelaram perdas de peso significativas entre mães que amamentaram e as que não amamentaram, e o mais curioso foi que, entre o nascimento e três meses após o parto, apenas o grupo com alimentação artificial apresentou perda de massa adiposa. Uma limitação desse estudo foi o reduzido número de mulheres estudadas em cada categoria de amamentação.

Outro estudo foi o desenvolvido por Potter et al. (1991) com 411 mulheres atendidas em uma clínica particular de Illinois (Estados Unidos da América), entre 1970 e 1980. As mulheres foram estudadas após o nascimento das crianças, com seis semanas e 12 meses após o parto, e divididas segundo a prática de aleitamento materno. As mães foram consideradas como tendo amamentado seus filhos se o aleitamento tivesse sido iniciado no hospital após o nascimento e se a amamentação tivesse sido mantida até a segunda observação, seis semanas depois do parto. Entre os principais resultados, observou-se que mães que praticaram aleitamento artificial tendiam a perder mais peso do que aquelas que amamentaram ao seio com seis semanas após o parto. Aos 12 meses depois do parto, o tipo de aleitamento não esteve relacionado com a perda de peso e, para o conjunto das mulheres, o peso médio ainda era de $2 \mathrm{~kg}$ a $5 \mathrm{~kg}$ maior que o peso pré-gestacional.

Uma limitação do estudo de Potter et al. (1991) foi o fato de que todas as informações foram coletadas de forma retrospectiva, não estando disponíveis dados de consumo de alimentos ou práticas de atividade física. Outra importante limitação foi o fato de que a lactação não pôde ser avaliada quanto à extensão de sua duração, e a definição de ter amamentado foi claramente insuficiente para que fosse observado impacto da amamentação na retenção de peso 12 meses após o parto, já que seis semanas é um tempo muito curto para duração do aleitamento.

Ainda na linha dos estudos que contestam o efeito protetor da lactação na perda de peso, destaca-se o de Schauberger et al. (1992). Esses autores investigaram diversos fatores que influenciaram a perda de peso no pós-parto em 795 mulheres que tiverem filho em uma maternidade de Wisconsin, entre 1989 e 1990. Em nenhuma das três entrevistas (duas semanas, seis semanas e seis meses), a perda de peso esteve associada à lactação, e a conclusão dos autores 
é de que o papel da lactação na perda de peso é muito pequeno nesse grupo de mulheres. Cabe ressaltar que, nesse estudo, a amamentação foi estudada de forma apenas periférica, não tendo sido devidamente aferida.

Segundo Lederman (1993), a ausência de associação entre lactação e perda de peso que certos estudos reportam pode estar relacionada à forma com que a lactação foi aferida, especialmente a intensidade, que em muitos estudos foi quantificada apenas de forma grosseira. Outras limitações dessas investigações envolvem número insuficiente de mulheres que amamentaram por mais de seis meses, não-exclusão de mulheres que estavam fazendo dieta e a ausência de grupos-controles bem pareados (Dewey et al., 1993).

Três importantes estudos que revelaram efeito protetor da amamentação na perda de peso merecem destaque.

No primeiro, o padrão de amamentação foi avaliado com base em um escore de lactação desenvolvido pelos autores para expressar a duração e a intensidade da lactação. Segundo esse escore, para cada mês de aleitamento exclusivo, eram computados quatro pontos, e, para cada mês de aleitamento misto, dois pontos. O somatório total variou entre 0 e 48 pontos, sendo considerado pelos autores como uma estimativa do total de energia despendida para produção de leite (Ohlin \& Rossner, 1990).

As mulheres que apresentaram mais tempo de amamentação exclusiva, mais tempo de amamentação mista, ou os mais altos escores de lactação tenderam a perder mais peso entre 2,5 e 12 meses após o parto, quando comparadas a mães que amamentaram menos, apesar de as análises de regressão não terem apresentado correlações significativas. Os resultados demonstraram que a perda de peso entre $2,5 \mathrm{e}$ 6 meses depois da gestação foi maior entre as mulheres com escore de lactação superior a vinte pontos. Por outro lado, 12 meses depois do parto, as diferenças não eram mais significativas. Outros resultados revelaram que $49 \%$ das mulheres com mais de quarenta pontos perderam mais de $3 \mathrm{~kg}$ entre 2,5 e 12 meses depois do parto, em comparação com de $33 \%$ a $35 \%$ das que apresentaram escore inferior a quarenta pontos. A relação entre lactação e perda de peso no pós-parto foi surpreendentemente fraca, apesar de seu maior efeito ocorrer entre 2,5 e 6 meses após o parto.

Dewey et al. (1993) analisaram o padrão de perda de peso durante 24 meses após o parto em 46 mulheres americanas que participaram do Estudo DARLING (Davies Area Research on Lactation, Infant Nutrition and Growth) e ama- mentaram por mais de 12 meses e em 39 mães que amamentaram por menos de três meses. No primeiro mês pós-parto, os dois grupos apresentaram o mesmo peso, no entanto, aos seis meses após o parto, a média de peso do grupo de mães que amamentaram 12 meses ou mais era aproximadamente $2,8 \mathrm{~kg}$ menor, ao passo que 12 meses depois do parto essa diferença foi de $3,2 \mathrm{~kg}$. No primeiro ano pós-parto, as mães que amamentaram por mais tempo perderam $4,4 \mathrm{~kg}$, e as que amamentaram menos de três meses perderam apenas $2,4 \mathrm{~kg}$. O padrão de perda de peso entre os grupos de mães demonstrou claramente que, nos três primeiros meses pós-parto, a perda foi semelhante, porém, entre três e seis meses, as diferenças foram altamente marcantes e significativas. Esses resultados indicam que as mulheres devem amamentar por mais de seis meses, se elas esperam que a lactação aumente a perda de peso.

Um dos estudos mais recentes sobre o tema foi desenvolvido com 110 mulheres americanas entre vinte e quarenta anos, recrutadas em ambulatórios de obstetrícia na cidade de Ann Arbor (Janney et al., 1997). Trata-se de um dos estudos cujo processo analítico é dos mais refinados. A metodologia empregada pelos autores envolveu modelos de regressão longitudinal, que permitem incluir co-variáveis tempodependentes ou não. A duração da lactação foi um importante preditor para retenção de peso no pós-parto. De uma forma geral, mulheres que amamentaram mais retiveram menos peso e atingiram seu peso pré-gestacional aproximadamente seis meses antes das mães que alimentaram seus filhos com formulados. Os resultados sugerem que a lactação influencia o padrão de perda de peso no pós-parto, no entanto o efeito da lactação foi suficientemente limitado para garantir a menor ênfase possível na amamentação enquanto método que minimize a retenção de peso no pós-parto (Janney et al., 1997).

Sem a pretensão de esgotar o assunto, que na realidade mantém-se em franca evolução, observa-se que os postulados de Robinson (1986) sobre mudanças na composição corporal durante a reprodução aplicam-se às mulheres durante a lactação. Mudanças no peso corpóreo e na massa adiposa impostos pela carga metabólica da lactação são altamente variáveis entre populações. A maioria dos estudos não reportou diferenças nas taxas de perda de peso entre mulheres lactantes e não lactantes, e apenas diferenças sutis foram observadas entre esses grupos (Butte \& Hopikinson, 1998).

Conforme já mencionado, as mudanças na composição corporal durante o período repro- 
dutivo derivam de uma seqüência complexa de estímulos neuroendócrinos e bioquímicos modificados pelo ambiente. Dessa forma, recomenda-se que, para alcançar níveis adequados de saúde e bem-estar, o objetivo nutricional seria produzir e manter um equilíbrio na composição corporal durante todo o período reprodutivo.

\section{Questões metodológicas sobre desenhos}

Um aspecto particularmente importante no desenvolvimento de estudos epidemiológicos sobre mudanças de peso no pós-parto referese às questões metodológicas específicas sobre o desenho. Não é propriamente um dos objetivos da presente revisão discutir minuciosamente questões metodológicas, mas aproveitase para apresentar alguns pontos de debate. Nesta seção, são discutidas algumas características e limitações de estudos epidemiológicos sobre o tema, como: ausência de grupos de comparação, inclusão ou não de adolescentes ( $<18$ anos), perdas de seguimento e questões relativas à forma de se aferir o peso pré-gestacional e, conseqüentemente, de se calcular o ganho de peso durante a gestação.

Um dos mais importantes pontos metodológicos envolve a utilização de grupos de comparação, que devem incluir mulheres não grávidas ou nulíparas. A adoção desses grupos permite isolar o efeito da paridade no aumento de peso, o que torna as estimativas sobre mudanças de peso no pós-parto mais acuradas. Apenas quatro estudos prospectivos incluíram grupos de comparação (Rookus et al., 1987; Smith et al., 1994; Williamson et al., 1994; Wolfe et al., 1997), já que essa estratégia metodológica nem sempre é factível, apesar de ser importante para aumentar a acurácia das estimativas.

Uma outra questão envolve a inclusão ou não de adolescentes na amostra. Acredita-se (Gunderson \& Abrams, 1999) que a inclusão de mulheres com menos de 18 anos no estudo possa gerar estimativas de ganho de peso gestacional com viés (sobreestimação) em virtude do ganho de peso relacionado com o crescimento físico. A magnitude do viés depende da proporção de adolescentes no estudo, e, em grandes amostras $(n>1.000)$, parece existir uma diminuição no viés decorrente da inclusão de adolescentes. É interessante observar, porém, que a não-inclusão de adolescentes pode fazer com que esse grupo seja muito pouco estudado, o que é ruim, especialmente em países on- de o número de adolescentes grávidas é relevante.

Um dos maiores problemas em estudos de acompanhamento consiste nas perdas de seguimento, com conseqüente diminuição na confiabilidade das generalizações. Estudos importantes, como o de Ohlin \& Rossner (1990) e de Schauberger et al. (1992), reportaram perdas de $38 \% 12$ meses após o parto e $41 \%$ três meses depois do parto, respectivamente. Perdas entre $20 \%$ e $25 \%$ podem comprometer a generalização dos achados. É interessante observar que, em algumas situações, essas são as únicas informações disponíveis.

Uma limitação importante comumente observada em estudos sobre retenção de peso consiste na utilização de dados de peso prégestacional reportados pela própria mulher. A utilização desse tipo de dado pode gerar viés nas estimativas de mudanças de peso no pósparto, apesar da alta correlação entre peso prégestacional reportado e o peso efetivamente aferido. A magnitude do viés varia de acordo com a massa corporal pré-gestacional, entre outros fatores (Gunderson \& Abrams, 1999). O grande problema é que os erros podem variar entre $1 \mathrm{~kg}$ e $5 \mathrm{~kg}$, o que pode superestimar a retenção de peso em mais de $100 \%$, ou mesmo interferir no cálculo do ganho de peso gestacional, um importante determinante para a retenção de peso no pós-parto. A forma mais acurada de se obter a informação seria medir o peso logo após a concepção, o que, em termos práticos, é muito pouco factível.

É importante salientar, por fim, que algumas dessas questões nem sempre são operacionalmente viáveis, especialmente em estudos nos quais a estrutura organizacional e financeira necessária não está sempre prontamente disponível.

\section{Conclusões}

Os principais fatores determinantes da retenção de peso em mulheres no pós-parto incluem o ganho de peso durante a gestação e a intensidade da lactação. Diversos estudos (Boardley et al., 1995; Gunderson \& Abrams, 1999; Ohlin \& Rossner, 1990; Parham et al., 1990; Schauberger et al., 1992;) revelaram que quanto maior o ganho de peso durante a gestação, maior a retenção de peso no pós-parto. Os achados em relação ao efeito protetor da amamentação sobre a retenção de peso são contraditórios. Alguns poucos estudos (Dewey et al., 1993; Greene et al., 1988; Janney et al., 1997; Kramer et al., 1993; Ohlin \& Rossner, 1990) revelaram efeito 
protetor, enquanto a grande maioria (Brewer et al., 1989; Dugdale \& Eaton-Evans, 1989; Manning-Dalton \& Allen, 1983; Naismith \& Ritchie, 1975; Potter et al., 1991; Schauberger et al., 1992) não identificou efeito significante. Diversos fatores, como a atividade física, o consumo de alimentos, idade de menarca e outros relacionados ao estilo de vida, não têm sido devidamente investigados.

No âmbito dos desenhos, os estudos sobre mudanças de peso no pós-parto devem preferencialmente utilizar grupos de comparação com mulheres em idade reprodutiva (não grávidas ou nulíparas) e incluir peso pré-gestacional aferido logo após a concepção. Esses estu-

\section{Agradecimentos}

O autor agradece à Professora Dra. Maria Helena D’Aquino Benício (Faculdade de Saúde Pública, Universidade de São Paulo) pelos comentários críticos e as sugestões feitas em versões anteriores do manuscrito. O autor também agradece à Fundação Universitária José Bonifácio e à Fundação de Amparo à Pesquisa do Estado do Rio de Janeiro, financiadoras do projeto de pesquisa. dos não devem incluir mulheres com menos de 18 anos e manter perdas de seguimento em taxas inferiores a $20 \%-25 \%$. Por outro lado, devem evitar amostras muito pequenas $(n<300)$; incluir tempo de seguimento superior a seis meses e proporcionar dados sobre ganho de peso gestacional. Os grandes desafios consistem, sobretudo, em superar as limitações metodológicas dos atuais estudos, enfocando, principalmente, questões ligadas ao efeito da genética nas mudanças de peso no pós-parto, assim como o papel de variáveis pouco investigadas até o momento, como a atividade física, o consumo de alimentos e a história obstétrica.

\section{Referências}

ABITBOL, M. M., 1969. Weight gain in pregnancy. American Journal of Obstetrics and Gynecology, 104:140-157.

ARROYO, P.; AVILA-RORAS, H.; FERNANDEZ, V.; CASANUEVA, E. \& GALVAN, D., 1995. Parity and the prevalence of overweight. International Journal of Gynecology and Obstetrics, 48:269-272.

BARBOSA, L.; BUTTE, N. F; VILLALPANDO, S.; WONG, W. W. \& SMITH, E. O., 1997. Maternal energy balance and lactation performance of Mesoamerindians as a function of body mass index. American Journal of Clinical Nutrition, 66:575-583.

BEMFAM (Sociedade Civil Bem-Estar Familiar no Brasil), 1997. Pesquisa Nacional sobre Demografia e Saúde. Rio de Janeiro: BEMFAM.

BILLEWICZ, W. Z. \& THOMSON, A. M., 1970. Body weight in parous women. British Journal of Preventive and Social Medicine, 24:97-104.

BOARDLEY, D. J.; SARGENT, R. G.; COKER, A. L.; HUSSEY, J. R. \& SHARPE, P. A., 1995. The relationship between diet, activity and other factors, and postpartum weight change by race. Obstetrics and Gynecology, 86:834-838. 
BREWER, M. M.; BATES, M. R. \& VANNOY, L. P., 1989. Postpartum changes in maternal weight and body fat depots in lactation vs non-lactating women. American Journal of Clinical Nutrition, 49:259265.

BROWN, J. E.; KAYE, S. A. \& FOLSON, A. R., 1992. Parity-related weight change in women. International Journal of Obesity, 16:627-631.

BUTTE, N. F. \& HOPKINSON, J. M., 1998. Body composition changes during lactation are highly variable among women. Journal of Nutrition, 128: 381S-385S.

COITINHO, D. C. A., 1998. A Influência da História Reprodutiva no Índice de Massa Corporal de Mulheres Brasileiras. Tese de Doutorado, São Paulo: Faculdade de Saúde Pública, Universidade de São Paulo.

DEWEY, K. G., 1998a. Maternal body composition, caloric restriction and exercise during lactation: An overview. Journal of Nutrition, 128:379S-380S.

DEWEY, K. G., 1998b. Effects of maternal caloric restriction and exercise during lactation. Journal of Nutrition, 128:386S-389S.

DEWEY, K. G.; HEINING, M. J. \& NOMMSEN, L. A., 1993. Maternal weight-loss patterns during prolonged lactation. American Journal of Clinical Nutrition, 58:162-166.

DUGDALE, A. E. \& EATON-EVANS, J., 1989. The effect of lactation and other factors on post-partum changes in body-weight and triceps skinfold thickness. British Journal of Nutrition, 61:149-153.

GREENE, G. W.; SMICIKLAS-WRIGHT, H.; SCHOLL, T. O. \& KARP, R. J., 1988. Post-partum weight change: How much of the weight gained in pregnancy will be lost after delivery? Obstetrics and Gynecology, 71:701-707.

GUNDERSON, E. P. \& ABRAMS, B., 1999. Epidemiology of gestacional weight gain and body weight changes after pregnancy. Epidemiologic Reviews, 21:261-275.

HELIOVARA, M. \& AROMAA, A., 1981. Parity and obesity. Journal of Epidemiology and Community Health, 35:197-199.

IM (Institute of Medicine), 1990. Subcommittee on Nutritional Status and Weight Gain during Pregnancy. Nutrition during Pregnancy. Washington, DC: National Academy of Sciences.

INAN (Instituto Nacional de Alimentação e Nutrição), 1991. Condições Nutricionais da População Brasileira: Adultos e Idosos. Brasília: INAN.

JANNEY, C. A.; ZHANG, D. \& SOWERS, M., 1997. Lactation and weight retention. American Journal of Clinical Nutrition, 66:1116-1124.

KEPPEL, K. G. \& TAFFEL, S. M., 1993. Pregnancy-related weight gain and retention: Implications of the 1990 Institute of Medicine Guidelines. American Journal of Public Health, 83:1100-1103.

KRAMER, F. M.; STUNKARD, A. J.; MARSHALL, K. A.; MCKINNEY, S. \& LIEBSCHUTZ, J., 1993. Breastfeeding reduces maternal lower-body fat. Journal of the American Dietetics Association, 93:429-433.

LEDERMAN, S. A., 1993. The effect of pregnancy weight gain on later obesity. Obstetrics and Gynecology, 82:148-55.

LEERMAKERS, E. A.; ANGLIN, K. \& WING, R. R., 1998. Reducing postpartum weight retention through a correspondence intervention. International Journal of Obesity, 22:1103-1109.

LOVELADY, C. A.; GARNER, K. E.; MORENO, K. L. \& WILLIAMS, J. P., 2000. The effect of weight loss in overweight, lactating women on the growth of their infants. New England Journal of Medicine, 342:449-453.

MANNING-DALTON, C. \& ALLEN, L. H., 1983. The effects of lactation on energy and protein consuption, postpartum weight change and body composition of well nourished North American women. Nutrition Research, 3:293-308.

McKEOWN, T. \& RECORD, R. G., 1957. The influence of reproduction on body weight in women. Journal of Endocrinology, 15:393-399.

MONTEIRO, C. A., 1999. Epidemiologia da obesidade. In: Obesidade (A. Halpern, A. F. G. Matos, H. L. Suplicy, M. C. Mancini \& M. T. Zanella, org.), pp. 1530, Porto Alegre: Lemos Editorial.

MONTEIRO, C. A. \& CONDE, W. L., 1999. A tendência secular da obesidade segundo estratos sociais: Nordeste e Sudeste do Brasil, 1975-1989-1997. Arquivo Brasileiro de Endocrinologia e Metabologia, 43:186-194.

MUSCATI, S. K.; GRAY-DONALD, K. \& KOSKI, K. G., 1996. Timing of weight gain during pregnancy: Promoting fetal growth and minimizing maternal weight retention. International Journal of Obesity and Related Metabolic Disorders, 20:526-532.

NAISMITH, D. J. \& RITCHIE, C. D., 1975. The effect of breast-feeding and artificial feeding on bodyweight, skinfold measurements and food intake of forty-two primiparous women. Proceedings of the Nutrition Society, 34:116A-117A.

NEWCOMBE, R. G., 1982. Development of obesity in parous women. Journal of Epidemiology and Community Health, 36:306-309.

OHLIN, A. \& ROSSNER, S., 1990. Maternal body weight development after pregnancy. International Journal of Obesity, 14:159-173.

OHLIN, A. \& ROSSNER, S., 1994. Trends in eating patterns, physical activity and socio-demographics factors in relation to postpartum body weight development. British Journal of Nutrition, 71:457-470.

OHLIN, A. \& ROSSNER, S., 1996. Factors related to body weight changes during and after pregnancy: The Stockholm pregnancy and weight development study. Obesity Research, 4:271-276.

PARHAM, E. S.; ASTROM, M. F. \& KING, S. H., 1990. The association of pregnancy weight gain with the mother's postpartum weight. Journal of the American Dietetics Association, 90:550-554.

PARKER, J. D. \& ABRAMS, B., 1993. Differences in postpartum weight retention between black and white mothers. Obstetrics and Gynecology, 81: 768-774.

POTTER, S.; HANNUM, S.; MCFARLIN, B.; ESSEXSORLIE, D.; CAMPBELL, E. \& TRUPIN, S., 1991. Does infant feeding method influence maternal pospartum weight loss? Journal of the American Dietetics Association, 91:441-446.

ROBINSON, J. J., 1986. Changes in body composition during pregnancy and lactation. Proceedings of the Nutrition Society, 45:71-80.

ROOKUS, M. A.; ROKEBRAND, P.; BUREMA, J. \& DEURENBERG, P., 1987. The effect of pregnancy 
on the body mass index 9 months postpartum in 49 women. International Journal of Obesity, 11: 609-618.

ROSSNER, S., 1992. Short communication: Pregnancy, weight cycling and weight gain in obesity. International Journal of Obesity, 16:147.

ROSSNER, S. \& OHLIN, A., 1995. Pregnancy as a risk factor for obesity: Lessons from Stockholm pregnancy and weight development study. Obesity Research, 3 (Sup. 2):67S-75S.

SAMPSELLE, C. M.; SENG, J.; YEO, S.; KILLION, C. \& OAKLEY, D., 1999. Physical activity and postpartum well-being. Journal of Obstetrics Gynecology and Neonatal Nursing, 28:41-49.

SCHAUBERGER, C. H.; ROONEY, B. L. \& BRIMER, L. M., 1992. Factors that influence weight loss in the puerperium. Obstetrics and Gynecology, 79:424429.

SCHOLL, T. O.; HEDIGER, M. L.; SCHALL, J. I.; ANCES, I. G. \& SMITH, W. K., 1995. Gestational weight gain, pregnancy outcome, and postpartum weight retention. Obstetrics and Gynecology, 86:423-427.

SHELDON, J. H. \& LONDON, M. D., 1949. Maternal obesity. Lancet, 2:6585-6587.

SICHIERI, R.; COITINHO, D. C. A.; LEÃO, M. M.; RECINE, E. \& EVEHART, J. E., 1994. High temporal, geographic, and income variation in body mass index among adults in Brazil. American Journal of Public Health, 85:690-694.
SMITH, D. E.; LEWIS, C. E.; CAVENY, J. L.; PERKINS, L. L.; BURKE, G. L. \& BILD, D. E., 1994. Longitudinal changes in adiposity associated with pregnancy. The Cardia study. JAMA, 271:1747-1751.

SOBAL, J. \& STUNKARD, A. J., 1989. Socioeconomic status and obesity: A review of the literature. Psychological Bulletin, 105:260-275.

SOWERS, M.; ZHANG, D. \& JANNEY, C. A., 1998. Interpregnancy weight retention patterning in women who breastfed. Journal of Maternal-Fetal Medicine, 7:89-94.

WILLIAMSON, D. F.; MADANS, J.; PAMUK, E.; FLEGAL, K. M.; KENDRICK, J. S. \& SERDULA, M. K., 1994. A prospective study of childbearing and 10year weight gain in US white women 25 to 45 years of age. International Journal of Obesity, 18:561-569.

WOLFE, W. S.; SOBAL, J.; OLSON, C. M.; FRONGILLO, E. A.; WILLIAMSON, D. F., 1997. Parity-associated weight gain and its modification by sociodemographic and behavioral factors: A prospective analysis in US women. International Journal of Obesity and Related Metabolic Disorders, 21:802810. 